\title{
ANALYTICAL SOLUTIONS OF NANO BOUNDARY LAYER FLOWS BY USING HE'S HOMOTOPY PERTURBATION METHOD
}

\author{
M.Khaki ${ }^{1}$, D.D.Ganji ${ }^{2, *}$ \\ 1. Departments of Mechanical Engineering, Islamic azad university-Sari branch- \\ Sari,Mazandaran,Iran \\ 2. Departments of Civil and Mechanical Engineering, University of Babol \\ Noshivani, Babol, Mazandaran, Iran
}

\begin{abstract}
The aim of this article is to examine nano boundary layer. The equations governing the flow on wedge are derived from continuity and Navier-Stoks equations. The boundary conditions for the governing equations are obtained from the nonlinear Navier slip condition. This boundary condition contains an arbitrary index parameter, denoted by $\mathrm{n}>0$, which appears in the coefficients of the ordinary differential equation to be solved. The coupled equations are transformed into one differential equation by similarity solution. The transformed equation is then solved by He's Homotopy perturbation method and an analytical solution will be achieved. The validity of results is verified by comparing results with existing numerical results. Results are presented for the $\mathrm{x}$ and $\mathrm{y}$ components of the velocity profiles. Results are in reasonable agreement with those provided by other numerical methods and demonstrate a good accuracy of the obtained analytical solutions.
\end{abstract}

Keyword: nano boundary, Homotopy Perturbation Method (HPM), Navier boundary

\section{INTRODUCTION}

In classical boundary layer theory, the condition of no-slip near solid walls is usually applied. Investigations show that the no-slip condition is no longer valid at the nano scale. Instead, a certain degree of tangential slip must be allowed .To describe the phenomenon of slip, Navier [1] introduced a boundary condition which states the component of the fluid velocity tangential to the boundary walls is proportional to the tangential stress. The linear Navier condition was first used for nano boundary layer by M.T.Matthews and J.M. Hill [2]. They used similarity solutions to produce one ordinary differential equation and then solved it with numerical methods. In numerical methods, stability and convergence should be considered to avoid divergence or inappropriate results. It's often so hard to gain an analytical solution for these kinds of problems which include nonlinear terms. In recent decades, analytical solutions have developed for nonlinear differential equations. One of these methods is Homotopy perturbation

\footnotetext{
* Corresponding author.Tel: +98 1113234 501; fax: +98 1113234201.

E-mail address: ddg_davood @yahoo.com (D.D.Ganji)
} 
method, (HPM). The Homotopy perturbation method (HPM) was introduced by Ji-Huan He $[3,6]$ for the first time. This method has been used by many authors such as Ganji in [7-10]. These papers are published to handle a wide variety of scientific and engineering applications such as: linear and nonlinear, homogeneous and inhomogeneous as well, because this method continuously deform a difficult problem into a simpler form, which is solvable. It's been shown by many authors that these methods provide improvements over existing numerical techniques. These methods give successive approximations of high accuracy of solutions. The aim of this paper is to analytically study the nano boundary flow on a wedge. The results are compared with numerical outcomes presented in pervious works. However, an analytical expression is more convenient for engineering calculation and is also the obvious starting step point for a better understanding.

\section{MATHEMATICAL MODEL}

In this paper, we shall study two-dimensional laminar flow on a wedge at nano scale. The governing equations are continuity and Navier Stoks equations[2]

$$
\begin{aligned}
& \frac{\partial u}{\partial x}+\frac{\partial v}{\partial y}=0, \\
& u \frac{\partial u}{\partial x}+v \frac{\partial u}{\partial y}=U \frac{d U}{d x}+\frac{\partial^{2} u}{\partial y^{2}},
\end{aligned}
$$

subject boundary conditions

$$
\begin{aligned}
& |u|=l\left(\left|\frac{\partial u}{\partial y}\right|\right)^{n} \text { and } \quad v=0 \text { at } y=0, \\
& u \equiv U(x)=a \cdot x^{m} \quad \text { as } y \rightarrow \infty .
\end{aligned}
$$

Here, $\mathrm{x}$ and $\mathrm{y}$, are the dimensionless Cartesian coordinates, $\mathrm{u}$ and $\mathrm{v}$ are the dimensionless velocity components along $\mathrm{x}$ and $\mathrm{y}$ axes, the constant $l>0$ is the dimensionless slip length and $U$ is a given external dimensionless inviscid velocity. The dimensionless parameters are scaled by $\mathrm{L}, \mathrm{R}$ and $U_{\infty}$ as following definitions [3],

$$
x=\frac{x^{*}}{L}, y=\frac{y^{*} \sqrt{R}}{L}, u=\frac{u^{*}}{U_{\infty}}, v=\frac{v^{*} \sqrt{R}}{U_{\infty}}, U=\frac{U^{*}}{U_{\infty}}, l=\frac{l^{*}}{U_{\infty}^{n-1} L^{n} R^{-n / 2}},
$$

where, $\left(x^{*}, y^{*}, u^{*}, v^{*}, U^{*}\right.$ and $\left.l^{*}\right)$, are dimensional values of parameters and $\mathrm{R}$ is Reynolds number and $U_{\infty}$ is the free stream velocity. The relationship between the wedge angle factor $\beta$ (Fig. 1) and the exponent $m$ is $\beta=\frac{2 m}{m+1}$. Using Lie symmetries analysis, Matthews and Hill [2], introduced the similarity transformation

$$
\eta=x^{-\frac{n-1}{3 n-2}} y \text {, }
$$


and the stream function $f(\eta)$ defined by

$$
\frac{u}{U}=f^{\prime}, \quad x^{\frac{2 n-1}{3 n-2}} \frac{v}{U}=\left(\frac{n-1}{3 n-2} \eta f^{\prime}-\frac{2 n-1}{3 n-2} f\right) .
$$

The relationship between $\mathrm{m}$ an $\mathrm{n}$ is $m=\frac{n}{3 n-2}$ where $n \neq 2 / 3$.With these transformations, (1) and (2) can be written as

$$
f^{\prime \prime \prime}+\frac{2 n-1}{3 n-2} f f^{\prime \prime}-\frac{n}{3 n-2}\left(f^{\prime 2}-1\right)=0 .
$$

The boundary conditions depend on the value of $n$. For $n \neq 2 / 3$ the boundary conditions are

$$
\begin{aligned}
& f=0, \quad\left|f^{\prime}\right|=l\left|f^{\prime \prime}\right|^{n} \text { at } \eta=0 \\
& f^{\prime} \rightarrow a \quad \text { as } \eta \rightarrow \infty .
\end{aligned}
$$

We applied HPM on (8) with boundary conditions (9) and (10) to achieve an analytical solution.

\section{APPLICATION OF HOMOTOPY PERTURBATION METHOD}

According to HPM, we can construct homotopy of (9) as follows

$$
H(f, p)=(1-\mathrm{p})\left(\left(f^{\prime \prime \prime}+2 f^{\prime \prime}\right)-\left(g_{0}^{\prime \prime \prime}+2 g_{0}^{\prime \prime}\right)\right)+\mathrm{p}\left(f^{\prime \prime \prime}+\frac{2 n-1}{3 n-2} f f^{\prime \prime}-\frac{n}{3 n-2}\left(f^{\prime 2}-1\right)\right)=0 \text {. }
$$

Substituting $f=f_{0}+P f_{1}+P^{2} f_{2}+\ldots$ and $g_{0}=0$ into (11) and rearranging the resultant equation based on powers of p-terms, we have:

$$
\begin{aligned}
& P^{0}: f_{0}^{\prime \prime \prime}+2 f_{0}^{\prime \prime}=0, \quad f_{0}(0)=0,\left|f_{0}^{\prime}(0)\right|=l^{*}\left|f_{0}^{\prime \prime}(0)\right|^{n}, f_{0}^{\prime}(\infty)=1 \\
& P^{1}: f_{1}^{\prime \prime \prime}+2 f_{1}^{\prime \prime}+\frac{2 n-1}{3 n-2} f_{0} f_{0}^{\prime \prime}-2 f_{0}^{\prime \prime}-\frac{n}{3 n-2} f_{0}^{\prime 2}+\frac{n}{3 n-2}=0 \\
& f_{1}(0)=0,\left|f_{0}^{\prime}(0)+f_{1}^{\prime}(0)\right|=l *\left|f_{0}^{\prime \prime}(0)+f_{1}^{\prime \prime}(0)\right|^{n}, f_{1}^{\prime}(\infty)=0 \\
& P^{2}: f_{2}^{\prime \prime \prime}+2 f_{2}^{\prime \prime}+\frac{2 n-1}{3 n-2}\left(f_{0} f_{1}^{\prime \prime}+f_{1} f_{0}^{\prime \prime}\right)-2 f_{1}^{\prime \prime}-\frac{2 n}{3 n-2}\left(f_{0}^{\prime} f_{1}^{\prime}\right)=0 \\
& f_{2}(0)=0,\left|f_{0}^{\prime}(0)+f_{1}^{\prime}(0)+f_{2}^{\prime}(0)\right|=l *\left|f_{0}^{\prime \prime}(0)+f_{1}^{\prime}(0)+f_{2}^{\prime \prime}(0)\right|^{n}, f_{2}^{\prime}(\infty)=0 \\
& P^{3}: f_{3}^{\prime \prime \prime}+2 f_{3}^{\prime \prime}+\frac{2 n-1}{3 n-2}\left(f_{0} f_{2}^{\prime \prime}+f_{2} f_{0}^{\prime \prime}+f_{1} f_{1}^{\prime \prime}\right)-2 f_{2}^{\prime \prime}-\frac{n}{3 n-2}\left(2 f_{0}^{\prime} f_{2}^{\prime}+f_{1}^{\prime}\right)^{2}=0 \\
& f_{3}(0)=0,\left|f_{0}^{\prime}(0)+f_{1}^{\prime}(0)+f_{2}^{\prime}(0)+f_{3}^{\prime}(0)\right|=l *\left|f_{0}^{\prime \prime}(0)+f_{1}^{\prime}(0)+f_{2}^{\prime \prime}(0)+f_{3}^{\prime \prime}(0)\right|^{n}, f_{3}^{\prime}(\infty)=0 .
\end{aligned}
$$

$f(\eta)$ can be obtained by solving the (12) to (15). In the same manner, the rest of components were obtained using the maple package.

\section{RESULTS AND DISCUSSION}

The procedure that this paper adopted in the current investigation of the nano boundary layer problem on a wedge can be described as follows: 
By using similarity solution the coupled differential equations, (1) and (2), are reduced to (8). Equation (8) with the boundary conditions, (9), is solved by using HPM. In the reference [2], (8) for $n>1$, is solved by numerical method but using Homotopy perturbation method, we could produce analytical solution.

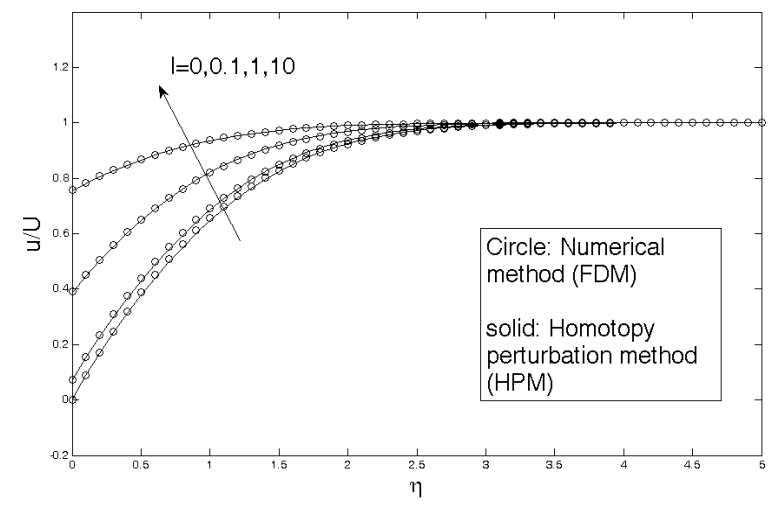

a

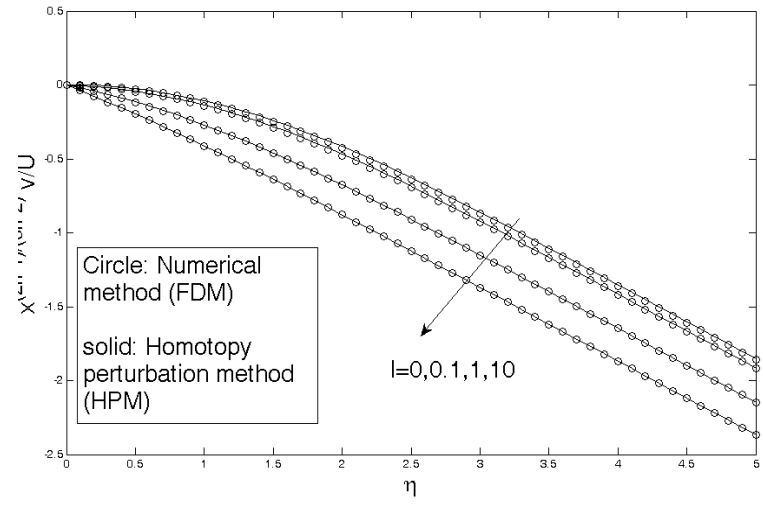

b

Fig. 2: Comparison between the FDM in reference [2] and HPM solutions for a: $\mathrm{u} / \mathrm{U}$ and $b: x^{(2 n-1) /(3 n-2)} v / U$ with $n=2$ and various values of 1

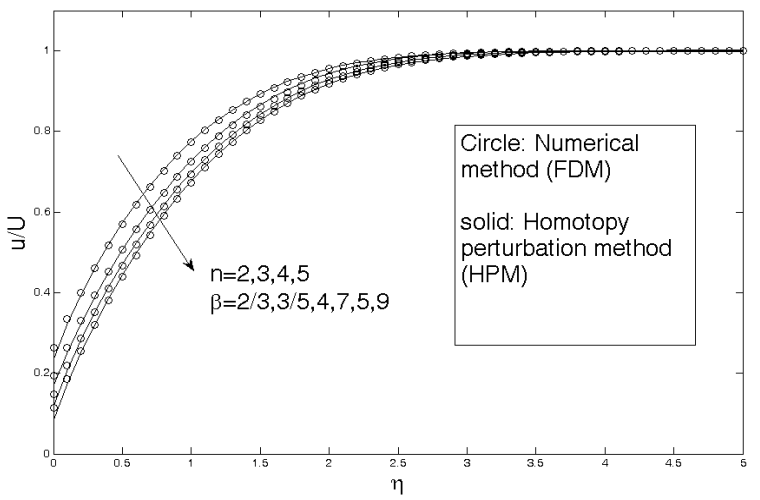

a

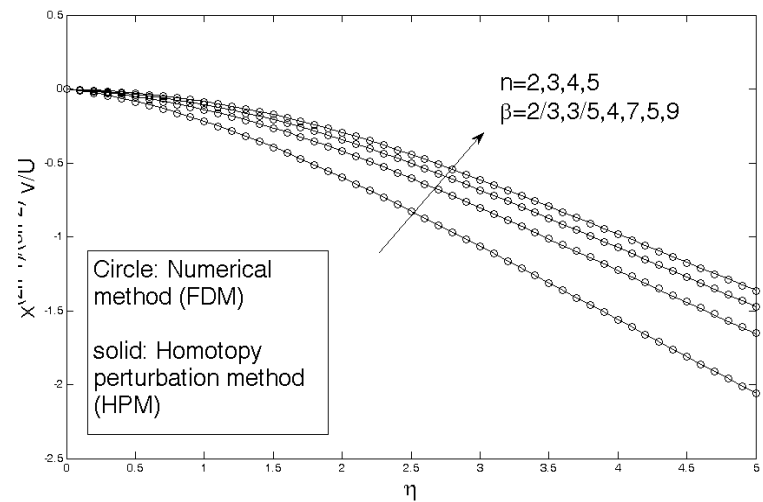

b

Fig. 3: Comparison between the FDM in reference [2] and HPM solutions for a: u/U and $b: x^{(2 n-1) /(3 n-2)} v / U$ with $l=0.5$ and various values of $n$

\section{CONCLUSION}

In this paper, we successfully applied homotopy perturbation method to solve a nonlinear differential equation with given boundary conditions for a nano boundary layer and showed the graphical results of $\mathrm{x}$ and $\mathrm{y}$ components of the velocity. Results were compared with numerical solution available in the literature using FDM and a very good agreement was observed. 
It is found that for fixed slip length the velocity components are reduced in magnitude as $n$ increases, while for fixed $n$ the velocity components are increased in magnitude as the slip length is increased. Also Fig. (3) show that the velocity components are increased in magnitude as the wage angle is increased. Outcomes prove the effectiveness and accuracy of HPM. The key-factor of this paper is based on analytically solving nano boundary layer problems.

\section{REFERENCES}

[1] C.L.M.H. Navier, Mémoire sur les lois du mouvement des fluids, Mém. Acad. Roy. Sci. Inst. France 6 (1823) 389-440.

[2] M.T.Matthews, J.M. Hill, Nano boundary layer equation with nonlinear Navier boundary condition, J. Math. Anal. Appl. 333 (2007) 381-400.

[3] J. H. He, Bookkeeping parameter in perturbation methods, Int. J. Non. Sc. Num. Sim. 2(3) (2001)257-264.

[4] J. H. He, Homotopy perturbation technique, Comp. Meth. App. Mech. Eng. 178, (1999) 257-262.

[5] J. H. He, A coupling method of a homotopy technique and a perturbation technique for non-linear problems, I. J. Non-Lin. Mech. 35 (2000)37-43.

[6] J. H. He, Homotopy perturbation method: a new nonlinear analytical technique, App. Math. Comp. 135 (2003) 73-79.

[7] D. D. Ganji, A. Sadighi, Application of He's Homotopy-perturbation Method to Nonlinear Coupled Systems of Reaction- diffusion Equations, Int. J. Nonlin. Sci. Num.7 (2006) 411-418.

[8] D. D. Ganji A. Sadighi, Solution of the Generalized Nonlinear Boussinesq Equation Using Homotopy Perturbation and Variational Iteration Methods, Int. J. Nonlin. Sci. Num.8 (2007) 435-444.

[9] M. Gorji, D. D. Ganji, S. Soleimani, New Application of He's Homotopy Perturbation Method, Int. J. Nonlin. Sci. Num.8 (2007) 319-328.

[10] H. Tari, D. D. Ganji, M. Rostamian, Approximate Solutions of K (2,2), KdV and Modified KdV Equations by Variational Iteration Method, Homotopy Perturbation Method and Homotopy Analysis Method, Int. J. Nonlin. Sci. Num.8 (2007) 203210. 